\title{
Centrifugal isolation of SARS-CoV-2: numerical simulation for purification of hospitals' air
}

\author{
Vahid Darvishi $^{1,2} \cdot$ Saeed Darvishi ${ }^{3} \cdot$ Marziyeh Bahrami-Bavani $^{4} \cdot$ Mahdi Navidbakhsh $^{1} \cdot$ Sasan Asiaei ${ }^{2}(\mathbb{C}$
}

Received: 30 March 2021 / Accepted: 7 June 2021 / Published online: 17 June 2021

(c) The Author(s), under exclusive licence to Springer-Verlag GmbH Germany, part of Springer Nature 2021

\begin{abstract}
Coronavirus and its spread all over the world have been the most challenging crisis in 2020. Hospitals are categorized among the most vulnerable centers due to their presumably highest traffic of this virus. In this study, centrifugal isolation of coronavirus is successfully deployed for purifying hospitals' air using air conditioners and ducts, suggesting an efficient setup. Numerical simulations have been used to testify the proposed setup due to the complexities of using experimental investigation such as high cost and clinical hazards of the airborne SARS-CoV-2 in the air. Results show that a 20-cm pipe with an inlet velocity of $4 \mathrm{~m} / \mathrm{s}$ constitutes the best choice for the separation and purification of air from the virus. The proposed scalable method also efficiently separates larger particles, but it can separate smaller particles too. Numerical results also suggest installing the air purifying system on the floor of the hospitals' room for maximum efficiency.
\end{abstract}

Keywords Coronavirus $\cdot$ Aerosol $\cdot$ Computational fluid dynamics $\cdot$ Air pollution prevention $\cdot$ Numerical simulation $\cdot$ Virus transmission

\section{Introduction}

SARS-CoV-2, which is also known by the name coronavirus 2 , has been the most challenging crisis in 2019-2020. This virus emerged in Wuhan City, China, and after a short time had spread. As of writing, about 79 million cases of infection were reported which more than one million of them are in Iran (World Health Organization 2020a).

Mahdi Navidbakhsh

mnavid@iust.ac.ir

Sasan Asiaei

asiaei@iust.ac.ir

1 Tissue Engineering and Biological Systems Research Laboratory, School of Mechanical Engineering, Iran University of Science and Technology, 16846 Tehran, Iran

2 Sensors and Integrated Bio-Microfluidics/MEMS Laboratory, School of Mechanical Engineering, Iran University of Science and Technology, 16846-13114 Narmak, Tehran, Iran

3 Mechanical Engineering Department, Babol Noshirvani University of Technology, 47148-71167 Babol, Iran

4 Department of Microbiology, Faculty of Biological Science, Alzahra University, Tehran, Iran
The main reason for coronavirus's power to spread is its ability to transmit airborne which is almost uncontrollable. Therefore, it is vital to understand the transmission routes of coronavirus to decrease its spreading. Based on WHO reports, SARS-CoV-2 is mainly transmitted by respiratory droplets. The transmission happens when two people or more are near each other (less than one meter distance (World Health Organization 2020b)). However, some studies examined and showed the transmission of viruses by aerosols. Some small particles called droplet nuclei which could be generated during daily activities also can be another way of airborne transmission of viruses (Judson and Munster 2019; Tellier et al. 2019). Other studies investigate transmission ways of SARS-CoV-2 from various perspectives such as aerosol generation from expiratory, fluid mechanics, and social distancing (Asadi et al. 2020; Mittal et al. 2020; Setti et al. 2020). A study by Van Doremalen et al. (2020) revealed that the SARS-CoV-2 was viable in the air after three hours . Many other studies have reported the detection of SARSCoV-2 genetic materials in the air. In Nebraska and Wuhan, air samples were gathered and researchers conducted SARS$\mathrm{CoV}-2$ nucleic acid tests on them. Results became positive in a patient room of Nebraska medical center and the hospital of Wuhan (Guo et al. 2020; Santarpia et al. 2020). SARS$\mathrm{CoV}-2$ was found in the air sample from a student health care 
center in Florida (Lednicky et al. 2020b). RT-PCR analysis of air samples approved the existence of SARS-CoV-2 in room air where infected patients were kept there in Singapore (Chia et al. 2020). Lednicky et al. showed in a study in 2020 that SARS-CoV-2 exists in samples of the surrounding of the patient (about 2-to $4.8 \mathrm{~m}$ away) (Lednicky et al. 2020a). The detection and existence of SARS-CoV-2 in air samples reveal that coronavirus bioaerosols existed at all the sampling locations including hospitals.

Since the air of public places like hospitals is contaminated by SARS-CoV-2, the need for purifying their air is highly sensed these days. In this study, an investigation on the separation of SARS-CoV-2 from the hospital's air and purifying it is done. Considering that experimental investigation in this area has its complexities such as high cost and unsafe procedure during the need for the existence of SARS-CoV-2 in the air, numerical investigations and simulations have to be used to help us developing and testifying our model. So, computational methods are deployed in this study. Such simulations have been done before by authors in other biological systems (Asiaei et al. 2019; Darvishi et al. 2019).

\section{Mathematical modeling of the separation medium}

The separation which has been used in this study is based on centrifugal force exerted on aerosols when they are passing the curved shape part of the cylinder. Particles feel a centrifugal force when they are passing a curve. The centrifugal force is perpendicular to the flow direction and raise by raising the mass of particles and as a consequence, heavier particles approach the outer wall of the curved cylinder at a higher rate. By using the fact that the mass of SARS-CoV-2 and aerosols carrying them is higher than air and its elements, centrifugal force is deployed for the separation of coronavirus from the air.

In the present work, parameters affecting the separation percentage have been justified to have a proper particle separation. These parameters are the inlet velocity of the airflow and the cylindrical pipe diameter. A range of particle diameters is also examined since there are different sizes of aerosols in the air that can be a carrier for coronavirus (Lee 2020).

To reduce the complexities of fabrication, a simple curved pipe channel is used in simulations. The schematic of the channel is depicted in Fig. 1. The main goal of this study is to have the least aerosols exiting half lower of the output of pipe. In this respect and by having exiting flow from the lower output of pipe as a flow that will return to the hospitals' room, we can design a system to reduce the pollution of SARS-CoV-2 and finally purify it.

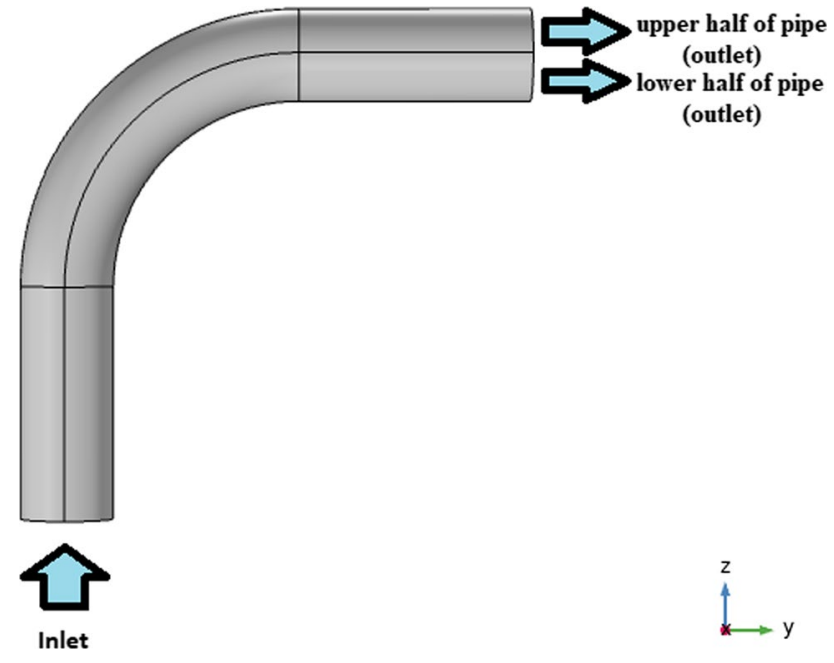

Fig. 1 Schematic of the channel used for SARS-CoV-2 separation

The geometry of the model consists of two straight and a $90^{\circ}$ cylindrical pipes. Each straight pipe has a $50 \mathrm{~cm}$ length and $20 \mathrm{~cm}$ diameter and curved pipe bend with a $50 \mathrm{~cm}$ radius of curvature (Fig. 1). In the beginning, the geometrical parameters are tuned using CFD simulations to make the separation of SARS-CoV-2 from air happen. In the next step, the geometry parameters are fixed and other hydrodynamic and physicochemical parameters are tuned to examine the percentage of separation in different conditions and maximize it.

To investigate the behavior of particles, two definitions can be introduced:1. purification percentage and 2. ratio of aerosols exit from the lower half of pipe to the total number of aerosols reach the output. Purification percentage represents the percentage of aerosols that are filtered in the mentioned channel and can be obtained by (Total number of aerosols inter the pipe - Total number of aerosols exit from the lower half of pipe) division to (Total number of aerosol inter the pipe) or Total number of arersols inter the pipe - Total number of aerosols exit from the lower half of the pipe. The total number of aerosols exit from the lower half of pipe division to the total number of aerosols reaches the output cross 100 (this percentage will be expressed by $\alpha$ in the following) which also can be used to describe and investigate the effects of centrifugal force on aerosols.

\subsection{Governing equations and effective forces}

In this simulation, there is a continuous phase and the velocity field could be described by Reynolds-averaged Navier-Stokes (RANS) equation and continuity equation represented in Eqs. 1 and 2. By having high flow rates and as a consequence high Reynolds number, the flow field 
exhibits small eddies and at least for the model presented in this study, the temporal and spatial scales of the oscillations become so small. In such a situation, it is unfeasible to resolve them using the Navier-Stokes equations computationally. By having this flow regime, RANS formulation can be used. RANS formulation is based on the fact that the flow field experiences small, local oscillations over time and so can be treated in a time-averaged sense (Tian and Ahmadi 2007) (Wilcox 2006).

$$
\begin{aligned}
\rho(u \cdot \nabla) u= & \nabla \cdot\left[-p I+\left(\mu+\mu_{T}\right)\left(\nabla u+(\nabla u)^{T}\right)\right. \\
& \left.-\frac{2}{3}\left(\mu+\mu_{T}\right)(\nabla \cdot u) I-\frac{2}{3} \rho k I\right]+F
\end{aligned}
$$

$\nabla \cdot(\rho u)=0$

where $u$ and $p$ are the time-averaged velocity and pressure, respectively. $\rho, \mu, \mu_{\mathrm{T}}, I$, and $F$ are fluid density, fluid viscosity, turbulent viscosity, identity tensor, and external forces, respectively. $\varepsilon$ and $\mathrm{k}$ denote dissipation rate and turbulence kinetic energy, respectively.

Also, by knowing that the inlet velocity of flow in this study is in the range of $0.5-20 \frac{\mathrm{m}}{\mathrm{s}}$, the Mach number is in the range of 0.00145 and 0.0583 so the air that is considered as the fluid in this study can be considered as an incompressible flow (Anderson 2010). As a result, Eqs. 1 and 2 simplify to Eqs. 3 and 4, respectively:

$\rho(u \cdot \nabla) u=\nabla \cdot\left[-p I+\left(\mu+\mu_{T}\right)\left(\nabla u+(\nabla u)^{T}\right)\right]+F$.

$\rho \nabla \cdot(u)=0$.

It has to be noted that for solving the RANS equation, there is a need to have additional transport equations to introduce turbulence variables described in Eq. $3\left(\mu_{\mathrm{T}}\right)$. Examples of these additional equations are the turbulence kinetic energy and the dissipation rate which are described in Eqs. 5 and 6 (Wilcox 2006).

$$
\begin{aligned}
& \rho(u \cdot \nabla) k=\nabla \cdot\left[\left(\mu+\frac{\mu_{\mathrm{T}}}{\sigma_{\mathrm{k}}}\right) \nabla k\right]+P_{\mathrm{k}}-\rho \varepsilon \\
& \rho(u \cdot \nabla) \varepsilon=\nabla \cdot\left[\left(\mu+\frac{\mu_{T}}{\sigma_{k}}\right) \nabla \varepsilon\right]+C_{\varepsilon 1} \frac{\varepsilon}{k} P_{k}-C_{\varepsilon 2} \rho \frac{\varepsilon^{2}}{k}
\end{aligned}
$$

$$
\begin{gathered}
\tau_{\mathrm{p}}=\frac{4 \rho_{\mathrm{p}} d_{\mathrm{p}}^{2}}{3 \mu c_{\mathrm{D}} \operatorname{Re}_{\mathrm{r}}} \\
\operatorname{Re}_{\mathrm{r}}=\frac{\rho|u-v| d_{\mathrm{p}}}{\mu}
\end{gathered}
$$

The remaining auxiliary relations and closure coefficients

$$
\begin{aligned}
& \sigma_{\mathrm{k}}=1, \sigma_{\varepsilon}=1.3, C_{\varepsilon 1}=1.44, \\
& C_{\varepsilon 2}=1.92, C_{\mu}=0.09
\end{aligned}
$$

Due to the high flow rate in this study and as described the forces of buoyancy, gravity, and inertia (drag) affect the particle and accelerate it. Newton's second law for a particle is represented as follows (Minea 2015):

$\frac{\mathrm{d}\left(m_{\mathrm{p}} v\right)}{\mathrm{d}_{\mathrm{t}}}=\sum F$

By considering various forces that affect the particle, Eq. 9 can be turned to:

$\frac{\mathrm{d}\left(m_{\mathrm{p}} v\right)}{\mathrm{d}_{\mathrm{t}}}=F_{\mathrm{D}}+F_{\mathrm{g}}-F_{\mathrm{B}}$

where $m_{\mathrm{p}}, v, g, \rho$, and $\rho_{\mathrm{P}}$ denote particle mass, particle velocity, gravity, fluid density, and particle density, respectively. gravity force, and buoyancy force exerted on the particle, respectively.

\subsubsection{Drag force}

In high Reynolds turbulence flow, the drag force is obtained from standard drag correlations. Also and by using the discrete random walk model (DRW), the instantaneous velocity is modeled (Clift et al. 2005).

$F_{\mathrm{D}}=\frac{1}{\tau_{\mathrm{p}}} m_{\mathrm{p}}\left(u^{\prime}-v\right)$

$$
\begin{aligned}
& c_{\mathrm{D}}=f\left(\operatorname{Re}_{\mathrm{r}}\right)= \\
& \begin{cases}\log C_{\mathrm{D}}=-2.4571+2.555 w-0.9295 w^{2}+0.1049 w^{3} & \text { if } 1500<\operatorname{Re}_{r} \leq 1.2 \times 10^{4} \\
\log C_{\mathrm{D}}=-1.9181+0.637 w-0.0636 w^{2} & \text { if } 1.2 \times 10^{4}<\mathrm{Re}_{r} \leq 4.4 \times 10^{4}\end{cases}
\end{aligned}
$$


$u^{\prime}=u+\Delta u \quad$ and $\quad \Delta u=\zeta \sqrt{\frac{2 k}{3}} \quad$ and $\quad w=\log \operatorname{Re}_{\mathrm{r}}$

where $\tau_{\mathrm{p}}, d_{\mathrm{p}}, c_{\mathrm{D}}, \operatorname{Re}_{\mathrm{r}}, u, \Delta u, \zeta$ are particle velocity response time, particle diameter, drag coefficient, relative Reynolds number, the time-averaged velocity of the air, instantaneous fluid velocity, and normally distributed random number, respectively.

\subsubsection{Gravity force}

Gravity which is proportional to particles' mass has been considered. By considering aerosols spherical, the gravity force can be written as:

$F_{\mathrm{g}}=\frac{4}{3} \pi r_{\mathrm{p}}^{3} \rho_{\mathrm{p}} g$

where $r_{\mathrm{p}}$ and $g$ are particle radius and gravity acceleration, respectively.

\subsubsection{Buoyancy force}

The buoyancy force is defined as an upward force made by fluid pressure which opposes the gravity force (Minea 2015) and can be written as:

$F_{\mathrm{B}}=m_{\mathrm{p}} g \frac{\rho}{\rho_{\mathrm{P}}}$

By using Eqs. (3-11), the real-time total force on aerosols will be determined. The inlet velocity is around $1 \frac{\mathrm{m}}{\mathrm{s}}$, and the outlet discharges to the atmosphere. The boundary conditions are illustrated in Table 1.

A numerical model has been implemented using Multiphysics software, and the Lagrangian approach also has been used to observe the particle trajectory in the airflow.

\subsubsection{Rotating frame}

The force that aerosol experiences in a curved pipe include centrifugal, Coriolis, and Euler forces.

Centrifugal force is an inertial force that is formulated in a rotating frame of reference (RFoR) and can be written as (Landau and Lifshitz 2013):

$F_{\text {cen }}=m_{\mathrm{p}} \Omega \times(r \times \Omega)$

Table 1 Boundary conditions

\begin{tabular}{lllll}
\hline$x(\mathrm{~cm})$ & $y(\mathrm{~cm})$ & $z(\mathrm{~cm})$ & $u(\mathrm{~m} / \mathrm{s})$ & $P(\mathrm{pa})$ \\
\hline$-10<\mathrm{x}<10$ & $-10<y<10$ & 0 & $u=4$ & N/A \\
$-10<x<10$ & 100 & $90<z<110$ & N/A & $\mathrm{Pd}=0$ \\
\hline
\end{tabular}

$r=q-r_{\mathrm{bp}}$

where $\Omega, q$, and $r_{\mathrm{bp}}$ are angular velocity, particle position, and a center of rotation, respectively.

Coriolis force acts on objects which are in motion within an RFoR that rotates concerning an inertial frame and can be described as (Landau and Lifshitz 2013):

$F_{\text {cor }}=2 m_{\mathrm{p}} v \times \Omega$

where $v$ denotes velocity.

Euler force is the last inertial force that appears when a non-uniform RFoR is used to analyze the motion and can be written as (Landau and Lifshitz 2013):

$F_{\mathrm{cul}}=m_{\mathrm{p}} r \times \dot{\Omega}$

where $\dot{\Omega}$ denotes angular acceleration.

All these forces affect the motion of an object in a rotating system such as a curved pipe. Nevertheless, they only appear in rotating coordinate systems.

\section{Results and discussion}

By using numerical simulation, the real-time separation is determined. The COMSOL Multiphysics solved the differential equations which were constructed for the numerical model. Tetrahedral 3D elements were used for meshing, and the size of meshes is refined to achieve a 1 percent difference in the numerical results which are obtained of finer and extra finer mesh. A maximum absolute tolerance of $10^{-3}$ was used in the time dependence solver.

Our numerical model has been verified against the numerical and experimental results of (Zhao et al. 2008), with the same initial and boundary conditions and particle properties. The field velocity was calculated, and the refinement of meshes was continued till their reports and our results deviated below 1 percent from each other. The results of the validation are illustrated in Fig. 2.

The physical and mechanical properties used in this numerical study are illustrated in Table 2.

\subsection{The practicality of separation of SARS-CoV-2 by using centrifugal force}

The feasibility of using the centrifugal method for purification of the hospital's air from coronavirus is examined in the first step. As it is illustrated in Fig. 3a and by using a $90^{\circ}$ curved shape pipe, a total purification of $87 \%$ has been achieved. This purification happens in a situation that by having a straight pipe, purification and separation do not happen as it is shown in Fig $3 b$. 


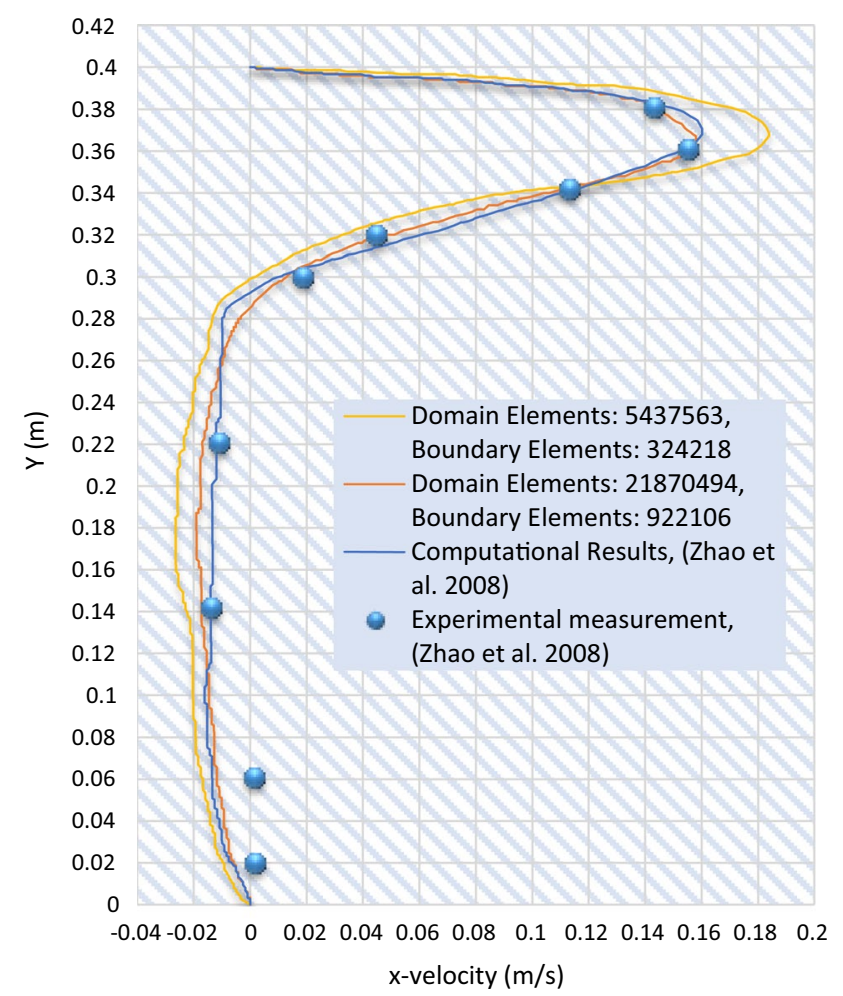

Fig. 2 Mesh independency and coincidence with (Zhao et al. 2008). The velocity field is calculated in the $\mathrm{x}$-direction

Table 2 Boundary conditions

\begin{tabular}{lll}
\hline Parameters & Air(Montgomery 1947) & $\begin{array}{l}\text { Aerosol(Joshi } \\
1988)\end{array}$ \\
\hline $\begin{array}{l}\text { Density }\left(\mathrm{kg} \mathrm{m}^{-3}\right) \\
\begin{array}{l}\text { Dynamic viscosity }(\mathrm{kg} \\
\left.\mathrm{m}^{-1} \mathrm{~s}^{-1}\right)\end{array}\end{array}$ & 1.204 & 1550 \\
\hline
\end{tabular}

\subsection{Pipe diameter}

Three different diameters are considered for designing and investigating proper pipe diameters. The range for this choice is based on commercial pipe diameters which are used for air conditioning (ToolBox 2005).

As it is illustrated in Fig. 4 and by having the minimum diameter for pipe, the maximum purification could be achieved. Another point that can play the main role in choosing the proper diameter is the time needed for every pipe to purify the room's air completely. By lowering the diameter from 30 to $10 \mathrm{~cm}$, the volume purification rate changes from 1.131 to $0.126 \frac{\mathrm{m}}{\mathrm{s}}$, respectively, considering $4 \frac{\mathrm{m}}{\mathrm{s}}$ as inlet flow rate. In other words, by considering a diameter of $10 \mathrm{~cm}$, it takes about 10 times longer than using $30 \mathrm{~cm}$ as the diameter of the pipe to purify a room's air. By considering these two parameters, the diameter of $20 \mathrm{~cm}$ is considered as pipe diameter to have more volume purification rate compared to $10 \mathrm{~cm}$ diameter and better separation and purification compared to $30 \mathrm{~cm}$ diameter.

\subsection{Inlet velocity}

To determine the role of inlet velocity and the effects of this parameter on the separation of coronavirus and percent of purification, six different velocities have been investigated. Choosing this range for inlet velocity is based on the suggestion of building engineering organization in which they suggested a range of 4-7 $\frac{\mathrm{m}}{\mathrm{s}}$ for inlet velocity of air conditioning to have the best circulation without making a disturbance in the room's air (ToolBox 2008). In addition to this range, lower and higher velocities are also considered to have a better understanding of the effects of this parameter on separation.

By raising the inlet velocity, more aerosols reach the output of the pipe. In the range between 0.5 and $10 \frac{\mathrm{m}}{\mathrm{s}}$, the total number of aerosols exit from the lower half of the pipe increases which means a decrease in the percentage of purification as shown in Fig. 5. For inlet velocities higher than $10 \frac{\mathrm{m}}{\mathrm{s}}$, this percentage is constant.

Results shown in Fig. 6 represent that although the total number of aerosols which they reach the output is increased and as a consequence, the purification percentage is reduced, a decrease happens in $\alpha$. This decrease in the ratio shows that by increasing the inlet velocity, aerosols passing the curved shape pipe experience higher centrifugal force.

\subsection{Different aerosols diameters}

Based on previous experimental investigations, ranges from 0.07 to $0.09 \mu \mathrm{m}$ are determined for the size of SARS-CoV-2 (Park et al. 2020; Wölfel et al. 2020). Lee BU introduced the range between 0.09 and $42 \mu \mathrm{m}$ for aerosols carrying coronavirus, which has $100 \%$ and $0.000001 \%$ viruses, respectively (Lee 2020). To investigate the percent of separation of each aerosol based on their diameter, a range between 1 and $40 \mu \mathrm{m}$ is considered since base numerical results show no changes for diameters lower than $1 \mu \mathrm{m}$. An inlet velocity of $4 \frac{\mathrm{m}}{\mathrm{s}}$ and pipe diameter of $20 \mathrm{~cm}$ are also considered based on previous sections.

Results show that by increasing the aerosols' diameter, the percent of purification increases as shown in Fig. 7. To put it in other words, bigger aerosols can be separated better than smaller aerosols. This phenomenon happens because centrifugal force increases by increasing the mass and mass of an aerosol increases by increasing its diameter. 

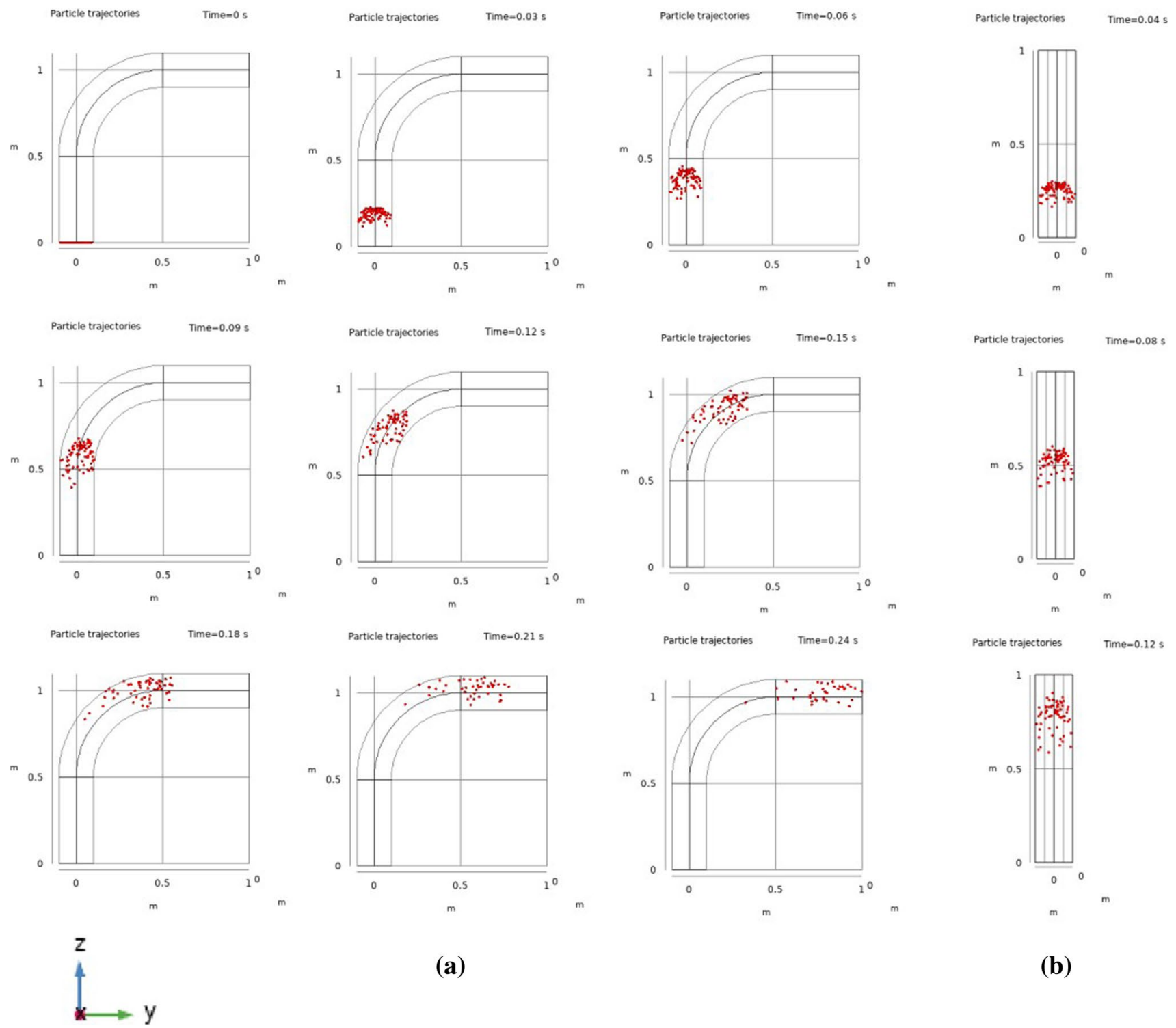

(a)

(b)

Fig. 3 Checking the feasibility of SARS-CoV-2 separation using centrifugal force. a Separation of aerosols happens in a curved pipe based on centrifugal force. $\mathbf{b}$ Separation does not happen in a straight pipe

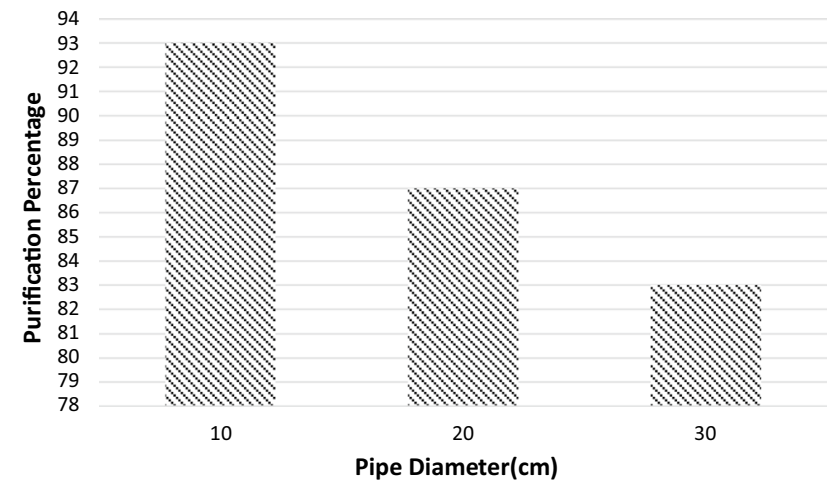

Fig. 4 Effects of pipe diameter on purification percentage

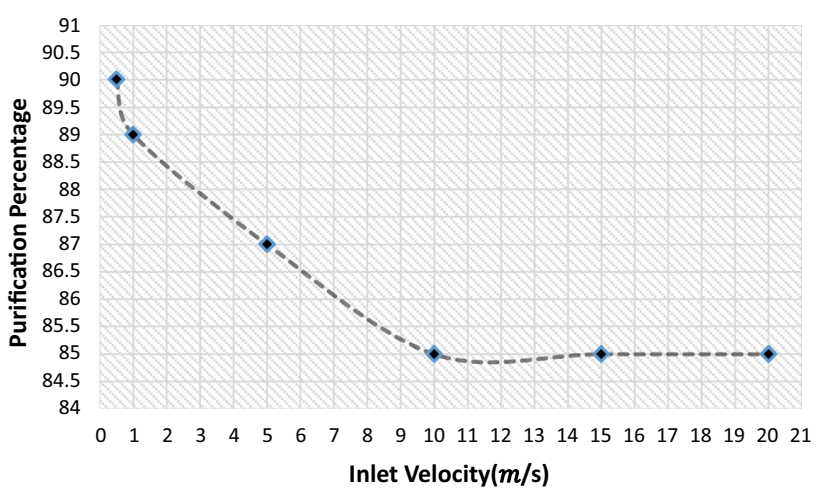

Fig. 5 Effects of inlet velocity on purification percentage 


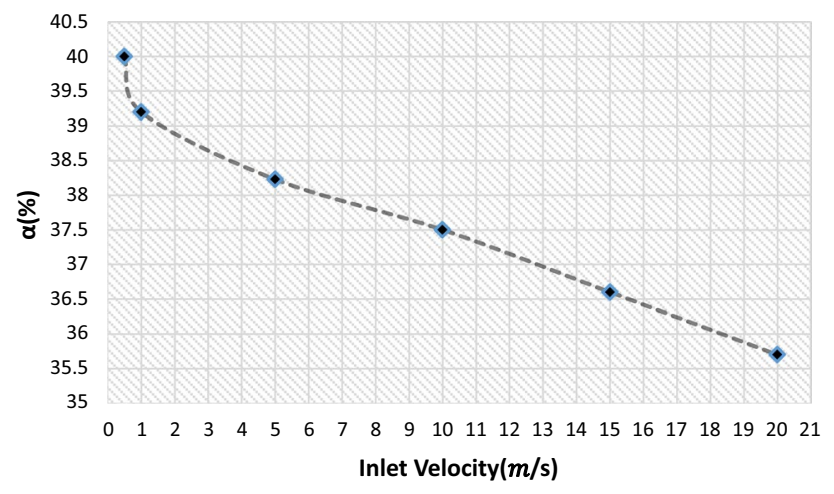

Fig. $6 \alpha$ number changes by increasing the inlet velocity

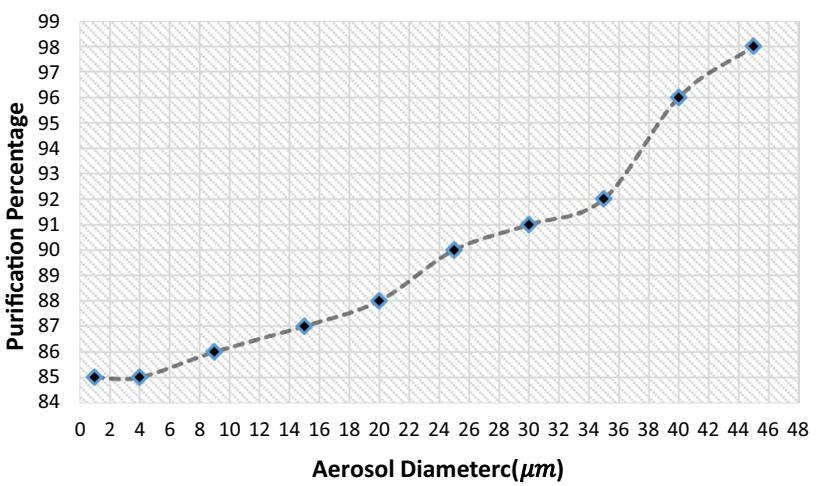

Fig. 7 Purification percentage of different aerosols

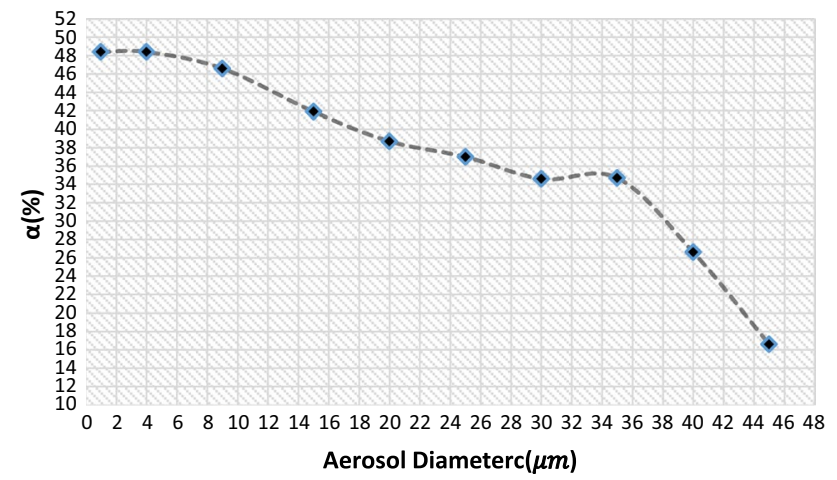

Fig. $8 \alpha$ number for different aerosols
As depicted in Fig. 8, the ratio of aerosols exit from the lower half of pipe to the total number of aerosols reaches the output of pipe which also decreases by increasing the aerosols' diameter.

As illustrated in Fig. 9, two different methods of using the proposed channel in hospitals' rooms (in the ceiling or on the floor) are investigated. This investigation is based on the direction of gravity force (agreeing with the direction of the centrifugal force in model $\mathrm{A}$ and opposite of the direction of centrifugal force in model B). Based on results, there is zero percent difference in separation with or without considering gravity. However, by knowing that gravity force can be neglected and so there is no difference between model $\mathrm{A}$ and model $B$ respecting this point of view, model $A$ is suggested by authors because aerosols are heavier than air and its components so their concentration is so much higher near the floor compared to the ceiling.

\section{Conclusion}

In this study, a system is designed and tested to purify hospitals' air from SARS-CoV-2. The centrifugal force has been successfully deployed for the separation of coronavirus and purification of air. Some parameters such as inlet velocity, pipe diameter, and aerosols' diameter that affect the percentage of purification are also investigated.

The result shows that centrifugal separation of SARSCoV-2 is a promising method to purifying the air of hospitals due to the high density of aerosols carrying coronavirus. Inlet velocity and pipe diameter can play an important role in this separation. In this study, the best inlet velocities and diameters considered $4 \frac{\mathrm{m}}{\mathrm{s}}$ and $20 \mathrm{~cm}$, respectively, to have proper separation and air conditioning but based on engineering designing can vary for different hospitals. Also, the best separation happens for bigger aerosols.

Result also indicates that buoyancy and gravity forces do not affect the separation due to the small dimension of aerosols and can be neglected. But in designing this system for separation, it is better to put the inlet of the channel on the floor (and not on the roof) since aerosols are heavier than air and its components and will come near to the ground after a while. 
Fig. 9 Two suggested duct setups

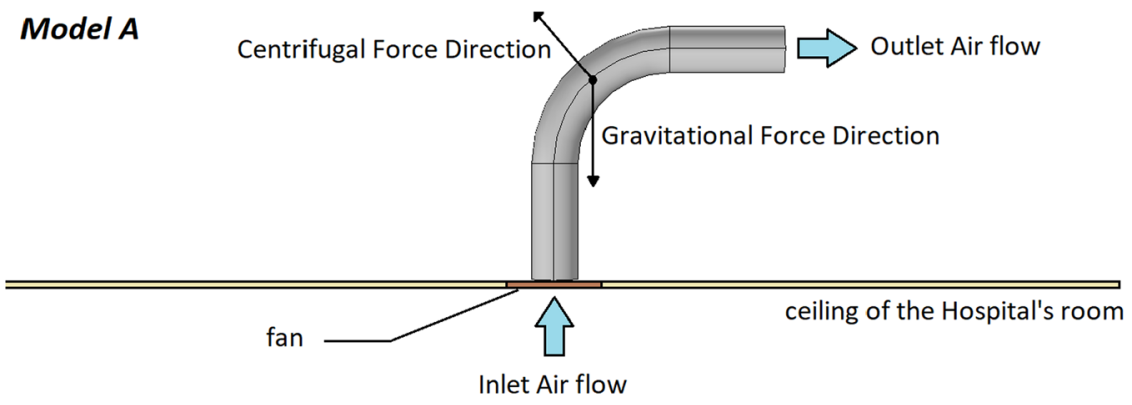

Model B

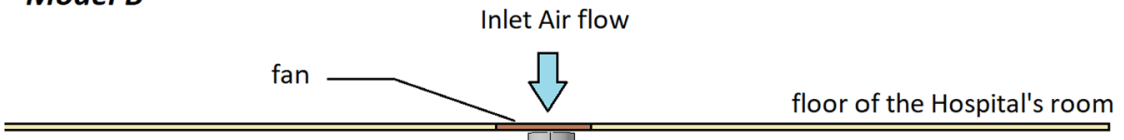

\section{References}

Anderson JD Jr (2010) Fundamentals of aerodynamics. Tata McGrawHill Education

Asadi S, Bouvier N, Wexler AS, Ristenpart WD (2020) The coronavirus pandemic and aerosols: Does COVID-19 transmit via expiratory particles?. 635-638. https://doi.org/10.1080/02786826.2020. 1749229

Asiaei S, Darvishi V, Davari MH et al (2019) Thermophoretic isolation of circulating tumour cells, numerical simulation and design of a microfluidic chip. J Therm Anal Calorim 137:831-839

Chia PY, Coleman KK, Tan YK et al (2020) Detection of air and surface contamination by SARS-CoV-2 in hospital rooms of infected patients. Nat Commun 11:1-7

Clift R, Grace JR, Weber ME (2005) Bubbles, drops, and particles. Courier Corporation

Darvishi V, Navidbakhsh M, Amanpour S (2019) Effects of temperature distribution in the tissue around the tumor on the quality of hyperthermia. In: 2018 25th National and 3rd international iranian conference on biomedical engineering (ICBME). IEEE, pp 1-6

Guo Z-D, Wang Z-Y, Zhang S-F et al (2020) Aerosol and surface distribution of severe acute respiratory syndrome coronavirus 2 in hospital wards, Wuhan, China, 2020. Emerg Infect Dis 26:10-3201

Joshi P V (1988) Density of atmospheric aerosol particles BT: atmospheric aerosols and nucleation. In: Wagner PE, Vali G (eds.). Springer, Berlin. pp 134-137

Judson SD, Munster VJ (2019) Nosocomial transmission of emerging viruses via aerosol-generating medical procedures. Viruses 11:940

Landau LD, Lifshitz EM (2013) Quantum mechanics: non-relativistic theory. Elsevier
Lednicky JA, Lauzard M, Fan ZH et al (2020a) Viable SARS-CoV-2 in the air of a hospital room with COVID-19 patients. Int J Infect Dis 100:476-482

Lednicky JA, Shankar SN, Elbadry MA et al (2020) Collection of SARS-CoV-2 virus from the air of a clinic within a university student health care center and analyses of the viral genomic sequence. Aerosol Air Qual Res 20:1167

Lee BU (2020) Minimum sizes of respiratory particles carrying SARSCoV-2 and the possibility of aerosol generation. Int J Environ Res Public Health 17:1-8. https://doi.org/10.3390/ijerph17196960

Minea AA (2015) Numerical studies on heat transfer enhancement in different closed enclosures heated symmetrically. J Therm Anal Calorim 121:711-720

Mittal R, Ni R, Seo J-H (2020) The flow physics of COVID-19. J Fluid Mech. https://doi.org/10.1017/jfm.2020.330

Montgomery RB (1947) Viscosity and thermal conductivity of air and diffusivity of water vapor in air. J Meteorol 4:193-196

Park WB, Kwon N-J, Choi S-J et al (2020) Virus isolation from the first patient with SARS-CoV-2 in Korea. J Korean Med Sci. https://doi. org/10.3346/jkms.2020.35.e84

Santarpia JL, Rivera DN, Herrera V, et al (2020) Transmission potential of SARS-CoV-2 in viral shedding observed at the University of Nebraska Medical Center. MedRxIV

Setti L, Passarini F, Gennaro G De, et al (2020) The potential role of particulate matter in the spreading of COVID-19 in Northern Italy: first evidence-based research hypotheses. MedRxiv, 2020.04. 11.20061713. https://www.medrxiv.org/content/101101/ 202004

Tellier R, Li Y, Cowling BJ, Tang JW (2019) Recognition of aerosol transmission of infectious agents: a commentary. BMC Infect Dis 19:101 
Tian L, Ahmadi G (2007) Particle deposition in turbulent duct flows - comparisons of different model predictions. J Aerosol Sci 38:377-397

ToolBox E (2005) Engineering toolbox. Circular ducts: dimensions. https://www.engineeringtoolbox.com/circular-ducts-d_1009.html

ToolBox E (2008) Engineering Toolbox. Duct sizing: equal friction method. https://www.engineeringtoolbox.com/equal-frictionmethod-d_1028.html

Van Doremalen N, Bushmaker T, Morris DH et al (2020) Aerosol and surface stability of SARS-CoV-2 as compared with SARS-CoV-1. N Engl J Med 382:1564-1567

Wilcox DC (2006) Turbulence Modeling for CFD Third Edition.. DCW Ind

Wölfel R, Corman VM, Guggemos W et al (2020) Virological assessment of hospitalized patients with COVID-2019. Nature $581: 465-469$
World Health Organization (2020a) WHO. Weekly epidemiological update, coronavirus disease 2019 (COVID-2019). https://www. who.int/publications/m/item/weekly-epidemiological-update--29-december-2020

World Health Organization (2020b) Transmission of SARS-CoV-2: implications for infection prevention precautions: scientific brief, 09 July 2020. World Health Organization

Zhao B, Yang C, Yang X, Liu S (2008) Particle dispersion and deposition in ventilated rooms: testing and evaluation of different Eulerian and Lagrangian models. Build Environ 43:388-397. https:// doi.org/10.1016/j.buildenv.2007.01.005

Publisher's Note Springer Nature remains neutral with regard to jurisdictional claims in published maps and institutional affiliations. 